Ann. Sci. forest., 1973, 30 (1), 63-81

\title{
ÉTUDE COMPLÉMENTAIRE DE LA PRÉSENCE DE CRISTAUX DE CARBONATE DE CALCIUM DANS LE BOIS DES PEUPLIERS EXISTENCE DE CINQ ZONES FONCTIONNELLES RECONNUES A PARTIR DE LEURS TENEURS EN PHOSPHORE
}

\author{
A. CLÉMENT et G. JANIN \\ Station de Recherches sur les Sols forestiers et la Fertilisation, \\ Station de Recherches sur la Qualité des Bois, \\ Centre national de Recherches forestières, I.N.R.A., \\ Champenoux 54370 Einville
}

RÉSUMÉ

Le présent rapport complète une étude parue précédemment sur la mise en évidence de cristaux de carbonate de calcium dans le bois des peupliers (Ann. Sci. forest., 1972, 29 (1), 67-105).

Après avoir examiné 28 espèces, nous retrouvons sans exception l'opposition déjà signalée entre les espèces de la section Leuce sans cristaux, et celles de toutes les autres sections Aigeiros, Leucoides, Tacamahaca et les hybrides entre ces dernières qui en contiennent. Ce caractère est indépendant de la nature du sol où se trouvent plantées ces espèces. l'eau.

Ces cristaux peuvent être facilement obtenus par broyage du bois et séparés par décantation dans

Il faut remarquer que dans certains cas, leur présence a une influence sur la technologie du débit de ces bois.

Par ailleurs, une analyse de tige portant sur le phosphore a été effectuée mètre par mètre et sur chaque section cerne par cerne sur un $P$. trichocarpa 'Fritzi-Pauley' de $25 \mathrm{~m}$ de hauteur; cette analyse a fait apparaître cinq zones fonctionnelles différentes : le bois de cime, la zone périphérique, l'aubier, le bois de cxur et le cerne central.

Ces distinctions qui se rapportent à la physiologie de l'arbre, faciliteront les études portant sur l'apparition du bois de cœur. 


\section{1. - INTRODUCTION}

Cette étude constitue une extension à nos recherches précédentes relatives à la présence de cristaux de carbonate de calcium dans le bois des peupliers (G. JANIN et A. CLÉMENT : Mise en évidence de cristaux de carbonate de calcium dans le bois des peupliers - Conséquences sur la répartition des ions minéraux liée à la duraminisation. Ann. Sci. forest., 1972, 30 (1), 67-105).

Elle rend compte des résultats complémentaires auxquels nous sommes parvenus concernant :

- d'une part la présence ou non de cristaux dans diverses espèces mise en évidence par radiographies aux rayons $\mathrm{X}$ et spectographies aux rayons $\mathrm{X}$.

- d'autre part les teneurs en phosphore dans les accroissements annuels d'un $P$. trichocarpa en liaison avec la duraminisation.

\section{2. - DÉTERMINATION QUALITATIVE}

\section{1. - Aspects dendrologiques}

Lors de nos premières observations, nous disposions de 7 espèces différentes comprenant 15 clônes ou variétés représentés par 234 arbres qui recouvraient les trois sections les plus importantes du genre Populus : Aigeiros, Tacamahaca et Leuce; malgré son étendue, un tel échantillonnage ne nous permettait cependant pas de conclure de façon certaine à la présence ou à l'absence de cristaux dans toutes les espèces de ces sections.

Pour avoir une confirmation de ce phénomène, nous avons procédé à l'examen de 28 espèces différentes de ces mêmes sections comprenant 51 clônes ou variétés avec au total 292 arbres.

Ces espèces provenaient toutes de populeta de collections ou de plantations d'origine connue : Alep (Syrie), Tokyo (Japon), Varsovie (Pologne), Velaine-sous-Amance (Meurthe-etMoselle), Villers-Cotterêts (Aisne) et Vineuil (Loir-et-Cher), cəci afin de recouper beaucoup mieux les déterminations et pour vérifier si, dans des sols et sous des climats différents, on pouvait observer un comportement identique pour un même cultivar.

\subsection{Description des sols.}

Les caractéristiques des sols des différents populeta sont les suivantes.

- Alep : $\mathrm{pH}=8,2$ — sol très calcaire — populetum irrigué — climat semi-aride.

- Tokyo : $\mathrm{pH}=4,5$ à 5,5 sol brun acide.

- Varsovie : $\mathrm{pH}=4,5$ - sol sableux reposant sur un matériel à sable grossier et moyen reposant sur de l'argile à galets.

- Velaine-sous-Amance : $\mathrm{pH}=5,5$ - sol à pseudo-gley développé sur un matériel limoneux reposant sur de l'argile.

- Villers-Cotterêts : $\mathrm{pH}=5$ - sol lessivé sur limon. 
- Vineuil $: \mathrm{pH}=6$ - sol alluvial léger et profond avec une nappe d'eau à profondeur variable entre 1 et 2 mètres.

Cette description sommaire présente un éventail assez complet des sols sur lesquels peuvent croitre les espèces étudiées, qui semble suffisant pour que la nature du terrain ne soit pas mise en cause en ce qui concerne la présence des cristaux.

\subsection{Tableaux des espèces.}

Les tableaux suivants rassemblent les espèces étudiées classées selon leur section, avec les indications concernant leur origine, le nombre d'individus examinés et la présence ou l'absence de cristaux :

TABLEAU 1

Section Leuce

\begin{tabular}{|c|c|c|c|c|}
\hline Espèces & $\begin{array}{l}\text { Cv. ou ssp. } \\
\text { ou var. }\end{array}$ & $\begin{array}{l}\text { Populetum } \\
\text { d'origine }\end{array}$ & 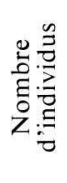 & 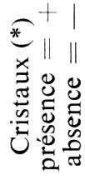 \\
\hline P. alba & $\begin{array}{l}\text { ' } \text { Roumi } \\
\text { - Roumi } \\
\text { no } 256 \\
\text { · } 5270 \\
\text { · } 5270 \\
\text { ' } 5376 \\
\cdot 5376\end{array}$ & $\begin{array}{l}\text { Alep (Syrie) } \\
\text { Vineuil } \\
\text { Varsovie (Pologne) } \\
\text { Vineuil } \\
\text { Velaine } \\
\text { Vineuil } \\
\text { Velaine }\end{array}$ & $\begin{array}{l}1 \\
1 \\
1 \\
1 \\
2 \\
1 \\
1\end{array}$ & $\begin{array}{l}- \\
- \\
-(2) \\
-(2)\end{array}$ \\
\hline$P . x$. canescens & $\begin{array}{l}\text { ' Megaleuce' M. L. B. } \\
\text { 'Megaleuce, M. L. B. } \\
\text { ' Bachofenii-5390 } \\
\text { 'Bachofenii-5390 }\end{array}$ & $\begin{array}{l}\text { Vineuil } \\
\text { Velaine } \\
\text { Vineuil } \\
\text { Velaine }\end{array}$ & $\begin{array}{l}1 \\
2 \\
1 \\
2\end{array}$ & $\begin{array}{l}-(2) \\
-(2)\end{array}$ \\
\hline$P$. grandidentata & ' S 5530', & $\begin{array}{l}\text { Vineuil } \\
\text { Velaine }\end{array}$ & $\begin{array}{l}1 \\
2\end{array}$ & - $_{(2)}$ \\
\hline P. tremula & $\begin{array}{l}\text { forme Gigas } \\
\text { ' DER 3, } \\
\text { ' DER 3, } \\
\text { ' MON } \\
\text { ' MON }\end{array}$ & $\begin{array}{l}\text { Vineuil } \\
\text { Vineuil } \\
\text { Velaine } \\
\text { Vineuil } \\
\text { Velaine }\end{array}$ & $\begin{array}{l}1 \\
1 \\
2 \\
1 \\
2\end{array}$ & $\begin{array}{l}- \\
-(2) \\
-(2)\end{array}$ \\
\hline P. tremuloüdes & $\begin{array}{l}\text { S } 5330 \\
\text { ' S } 5531\end{array}$ & $\begin{array}{l}\text { Vineuil } \\
\text { Velaine }\end{array}$ & $\begin{array}{l}1 \\
2\end{array}$ & - (2) \\
\hline P. tremula $x$ tremulö̈des & & Vineuil & 1 & - \\
\hline
\end{tabular}

(*) Le chiffre indiqué entre parenthèses dans ce tableau et dans les suivants se rapporte au nombre d'individus contenant $(+)$ ou ne contenant pas $(-)$ de cristaux pour la variété ou le clône étudié. 
TABLEAU 2

Section Aigeiros

\begin{tabular}{|c|c|c|c|c|}
\hline Espèces & $\begin{array}{l}\text { Cv. ou ssp. } \\
\text { ou var. }\end{array}$ & $\begin{array}{l}\text { Populetum } \\
\text { d'origine }\end{array}$ & 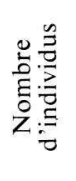 & 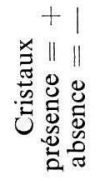 \\
\hline P. nigra & $\begin{array}{l}\text { 'Blane de Garonne, } \\
\text { ' Hamoui } \\
\text { 'Vert de Garonne } \\
\text { sinensis } \\
\text { no } 109\end{array}$ & $\begin{array}{l}\text { Vineuil } \\
\text { Alep (Syrie) } \\
\text { Vineuil } \\
\text { Vineuil } \\
\text { Varsovie (Pologne) }\end{array}$ & $\begin{array}{l}1 \\
1 \\
1 \\
1 \\
1\end{array}$ & $\begin{array}{l}+ \\
+ \\
+ \\
+ \\
+\end{array}$ \\
\hline$P$. deltoïdes & $\begin{array}{l}\text { 'SP 20-51-2, } \\
\text { 'Angulata de Chautagne, } \\
\text { monilifera' SP } 1091\end{array}$ & $\begin{array}{l}\text { Vineuil } \\
\text { Vineuil } \\
\text { Vineuil }\end{array}$ & $\begin{array}{l}1 \\
1 \\
1\end{array}$ & $\begin{array}{l}+ \\
+ \\
+\end{array}$ \\
\hline$P . x$. euramericana & 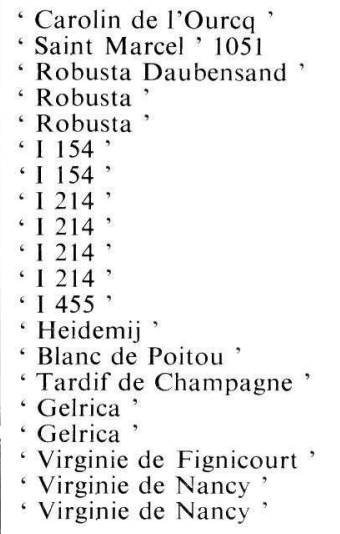 & $\begin{array}{l}\text { Vineuil } \\
\text { Vineuil } \\
\text { Vineuil } \\
\text { Vineuil } \\
\text { Velaine } \\
\text { Vineuil } \\
\text { Tokyo (Japon) } \\
\text { Tokyo (Japon) } \\
\text { Vineuil } \\
\text { Velaine } \\
\text { Villers-Cotterêts } \\
\text { Velaine } \\
\text { Vineuil } \\
\text { Vineuil } \\
\text { Vineuil } \\
\text { Velaine } \\
\text { Vineuil } \\
\text { Vineuil } \\
\text { Vineuil } \\
\text { Velaine }\end{array}$ & $\begin{array}{r}1 \\
1 \\
1 \\
1 \\
13 \\
1 \\
3 \\
3 \\
1 \\
23 \\
144 \\
1 \\
1 \\
1 \\
2 \\
6 \\
1 \\
1 \\
1 \\
4\end{array}$ & $\begin{array}{l}+ \\
+ \\
+ \\
+ \\
+(13) \\
+ \\
+(3) \\
+(3) \\
+ \\
+(23) \\
+(144) \\
+ \\
+ \\
+ \\
+(2) \\
+(6) \\
+ \\
+ \\
+ \\
+(4)\end{array}$ \\
\hline
\end{tabular}

\subsection{Observations et discussion.}

Ces tableaux nous permettent de faire les remarques suivantes :

- Le grand nombre d'espèces examinées et la concordance des résultats avec notre précédente étude nous autorisent à étendre à l'ensemble du genre nos conclusions sur l'absence de cristaux dans la section Leuce et leur présence dans toutes les autres (figures 1 et 2). Le nombre de ces cristaux peut être variable pour chaque arbre dans un même clône, exemple : I 214, fig. 3.

- La variété de la nature des sols d'où proviennent ces espèces nous indique que cet effet n'est pas lié au sol, mais qu'il relève de la physiologie particulière des arbres de chaque section.

- L'échantillonnage pratiqué sur la majeure partie de ces espèces sous forme de carottes de sondage, qui représentent une quantité réduite de bois de chaque arbre, suffit à montrer la régularité de la répartition des cristaux quand ils existent. 
TABLEAU 3

Section Tacamahaca

\begin{tabular}{|c|c|c|c|c|}
\hline Espèces & $\begin{array}{l}\text { Cv. ou ssp. } \\
\text { ou var. }\end{array}$ & $\begin{array}{l}\text { Populetum } \\
\text { d'origine }\end{array}$ & 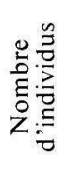 & 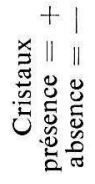 \\
\hline$P$. angustifolia & & Vineuil & 1 & + \\
\hline$P$. candicans & & Vineuil & 1 & + \\
\hline P. koreana & & Vineuil & 1 & + \\
\hline P. laurifolia & & Vineuil & 1 & + \\
\hline P. maximowiczii & & Vineuil & 1 & + \\
\hline P. simonii & & Vineuil & 1 & + \\
\hline P. szechuanica & & Vineuil & 1 & + \\
\hline P. tacamahaca & & Vineuil & 1 & + \\
\hline P. trichocarpa & $\begin{array}{l}\text { 'Fritzi Pauley } \\
\text { - Fritzi Pauley } \\
\text { ' Groenendael } \\
\text { 'Tervuren }\end{array}$ & $\begin{array}{l}\text { Velaine } \\
\text { Vineuil } \\
\text { Vineuil } \\
\text { Vineuil }\end{array}$ & $\begin{array}{r}25 \\
2 \\
1 \\
1\end{array}$ & $\begin{array}{l}+(25) \\
+(2) \\
+ \\
+\end{array}$ \\
\hline P. yunnanensis & & Vineuil & 1 & + \\
\hline P. maximowiczii $x$ trichocarpa & 'Androscoggin' & Vineuil & 1 & + \\
\hline
\end{tabular}

TABLEAU 4

Section Leucoides

\begin{tabular}{|c|c|c|c|c|}
\hline Espèces & $\begin{array}{l}\text { Cv. ou ssp. } \\
\text { ou var. }\end{array}$ & $\begin{array}{l}\text { Populetum } \\
\text { d'origine }\end{array}$ & 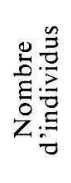 & 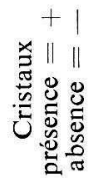 \\
\hline P. lasiocarpa & & Vineuil & 1 & + \\
\hline
\end{tabular}


TABLEAU 5

Hybrides entre sections

\begin{tabular}{|c|c|c|c|c|}
\hline Espèces & $\begin{array}{l}\text { Cv. ou ssp. } \\
\text { ou var. }\end{array}$ & $\begin{array}{l}\text { Populetum } \\
\text { d'origine }\end{array}$ & $\begin{array}{l}\text { Nombre } \\
\text { d'individus }\end{array}$ & $\begin{array}{c}\text { Cristaux } \\
\text { présence }=+ \\
\text { absence }=-\end{array}$ \\
\hline $\begin{array}{l}P . \text { acuminata } \\
\text { (angustifolia } x \text { sargentii) }\end{array}$ & & Vineuil & 1 & + \\
\hline $\begin{array}{l}\text { P. certinensis } \\
(\text { nigra ' italica ' } x \text { laurifolia })\end{array}$ & & Vineuil & 1 & + \\
\hline $\begin{array}{l}\text { P. generosa } \\
\text { (deltoüdes x trichocarpa) }\end{array}$ & & Vineuil & 1 & + \\
\hline $\begin{array}{l}P . \text { jackii } \\
\text { (deltoïdes } x \text { balsamifera) }\end{array}$ & & Vineuil & 1 & + \\
\hline P. manitobensis & & Vineuil & 1 & + \\
\hline P. maximowiczii $x$ berolinensis & ' Oxford & Vineuil & 1 & + \\
\hline P. nigra $x$ alba & & Varsovie & 1 & + \\
\hline
\end{tabular}

— Nous avons examiné un hybride $P$. nigra + 수 $\times P$. alba $\hat{\circ}$ qui contenait des cristaux. Dans cettehybridation, tous les individus avaient les caractères morphologiques de la mère. Nous nous trouvons donc, soit dans un cas de parthénocarpie (sans aucun gène paternel), soit dans un cas d'hybride vrai où les caractères de la section Aigeiros prédominent avec entre autres la présence de cristaux. 


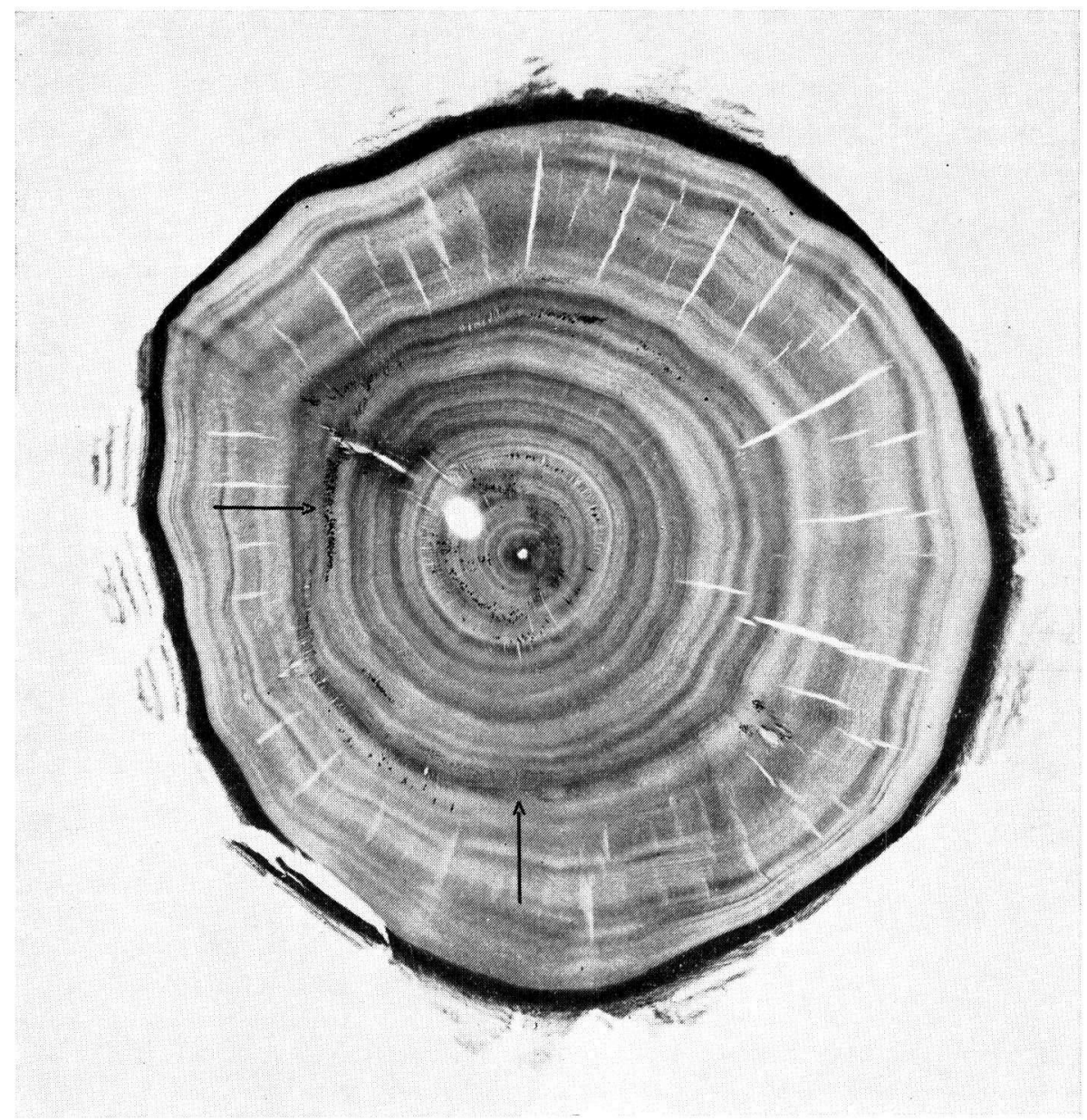

FIG. 1. - Positif de radiographie de P. nigra (grandeur nature) présentant des inclusions typiques de cristaux

- Sur cette image inversée, les cristaux apparaissent sous forme de points très noirs localisés dans les vaisseaux (flèche).

- Les radiographies prises dans les sections Tacamahaca, Leucoides et les hybrides entre ces sections présentent le même aspect.

FIG. 1. - X-ray radiograph positive print of $\mathrm{P}$. nigra (original size) showing typical mineral streaks of crystals

- On this inverted picture crystals appear within vessels like black points (arrow).

- The radiographs obtained in sections Tacamahaca, Leucoides and hybrids of these sections look as this one. 


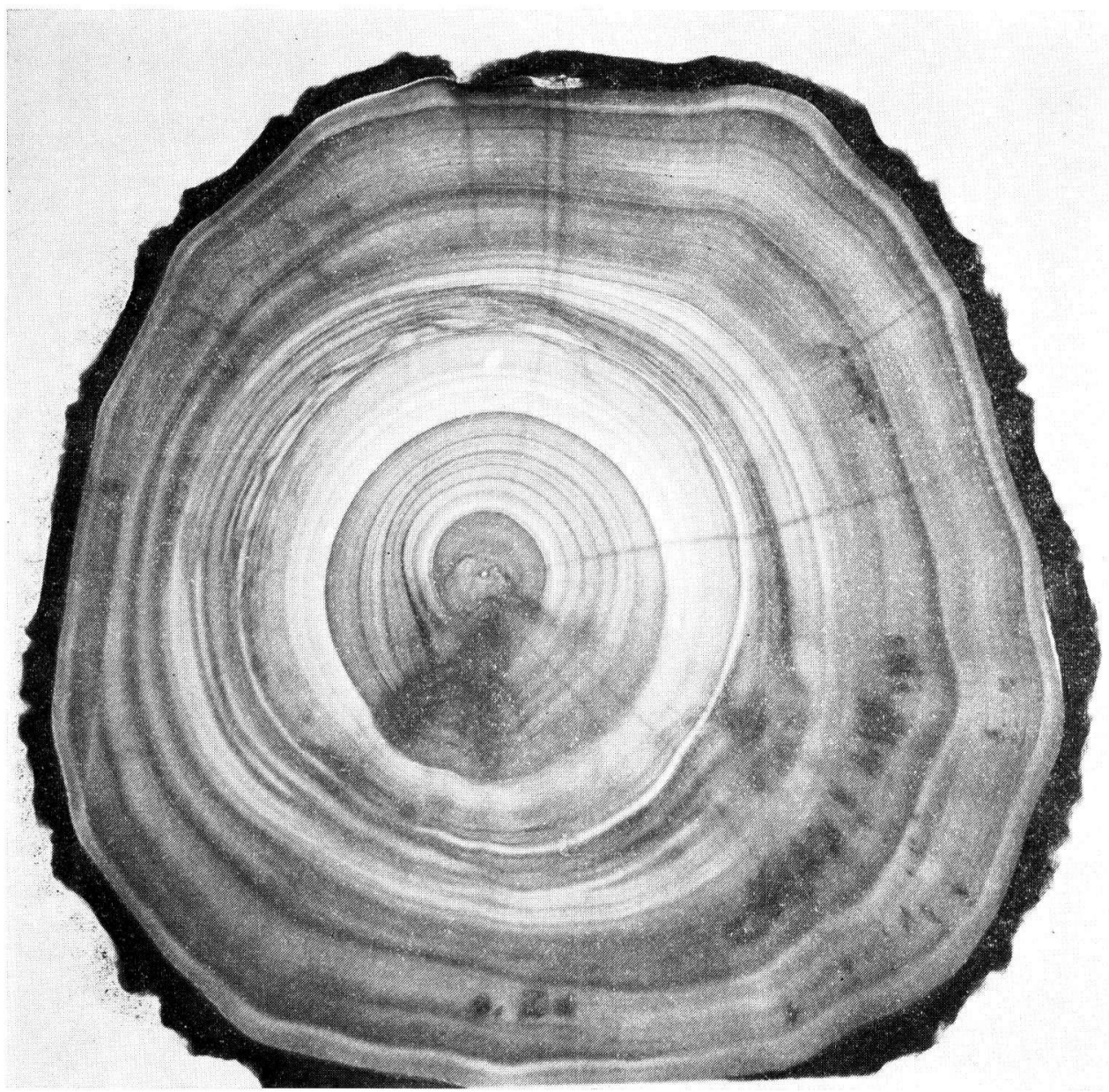

FIG. 2. - Positif de radiographie de P. alba 'Roumi' (grandeur nature)

- L'absence de cristaux est caractéristique de toute la section Leuce.

- Les tâches et les traînées grises sont, soit des défauts du bois, soit des accumulations de produits organiques.

FIG. 2. - X-ray radiograph positive print of P. alba 'Roumi' (original size)

- The absence of crystals is characteristic of the whole section Leuce.

- Spots and gray trails are wood defects or organic compounds accumulation. 

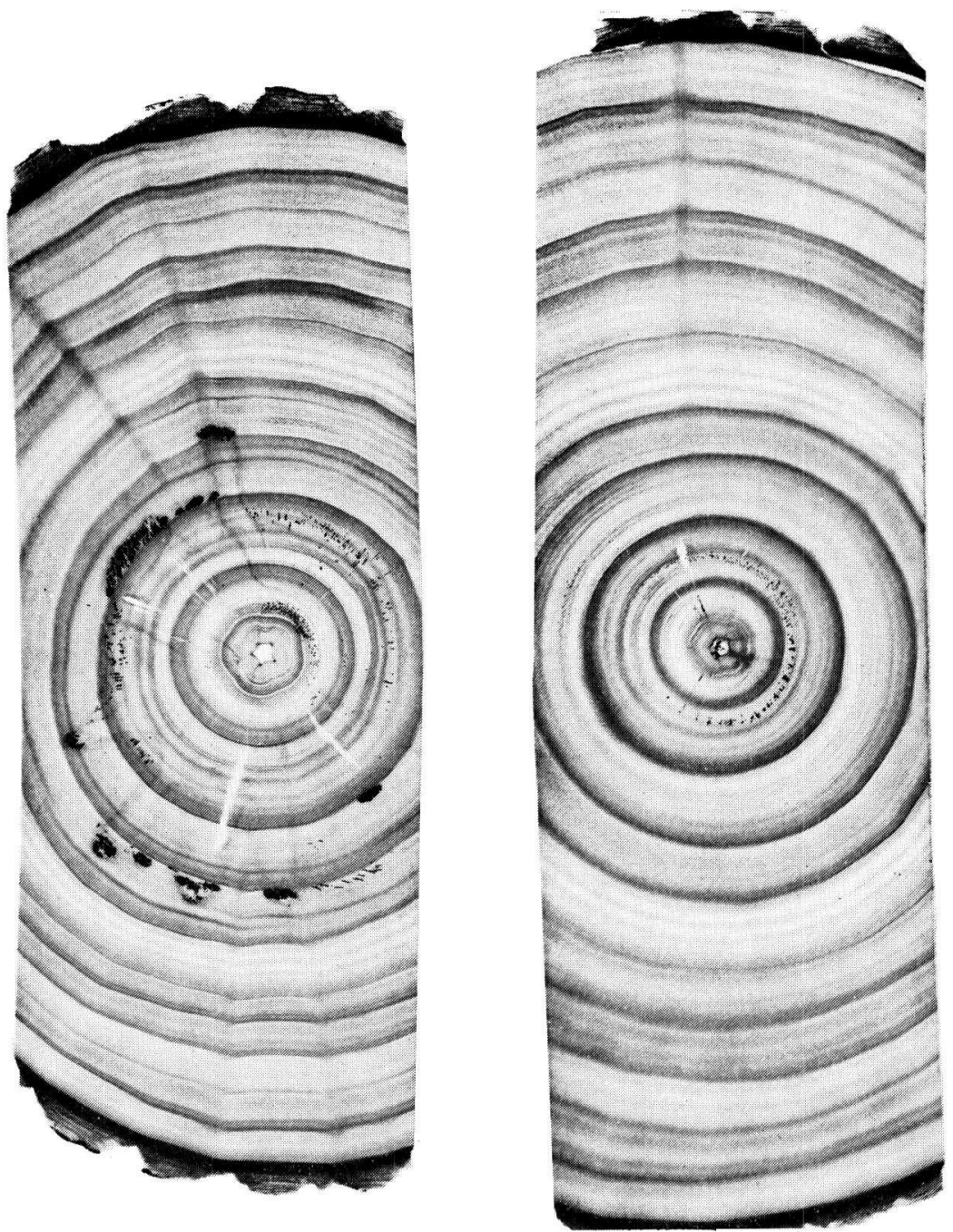

FIG. 3. - Positif de radiographie de P. x euramericana I 214 montrant la variabilité de l'importance des dépôts dans le même clône sur deux arbres différents

FIG. 3. - X-ray radiograph positive print of $\mathrm{P} x$ euramericana $\mathrm{I} 214$ showing the variability of deposits in the same section on two differents trees

\section{2. - Aspects cristallographiques}

\subsection{Obtention des cristaux purs.}

Des progrès ont été réalisés depuis notre précédent travail dans l'obtention de cristaux purs en grande quantité (figure 4). 

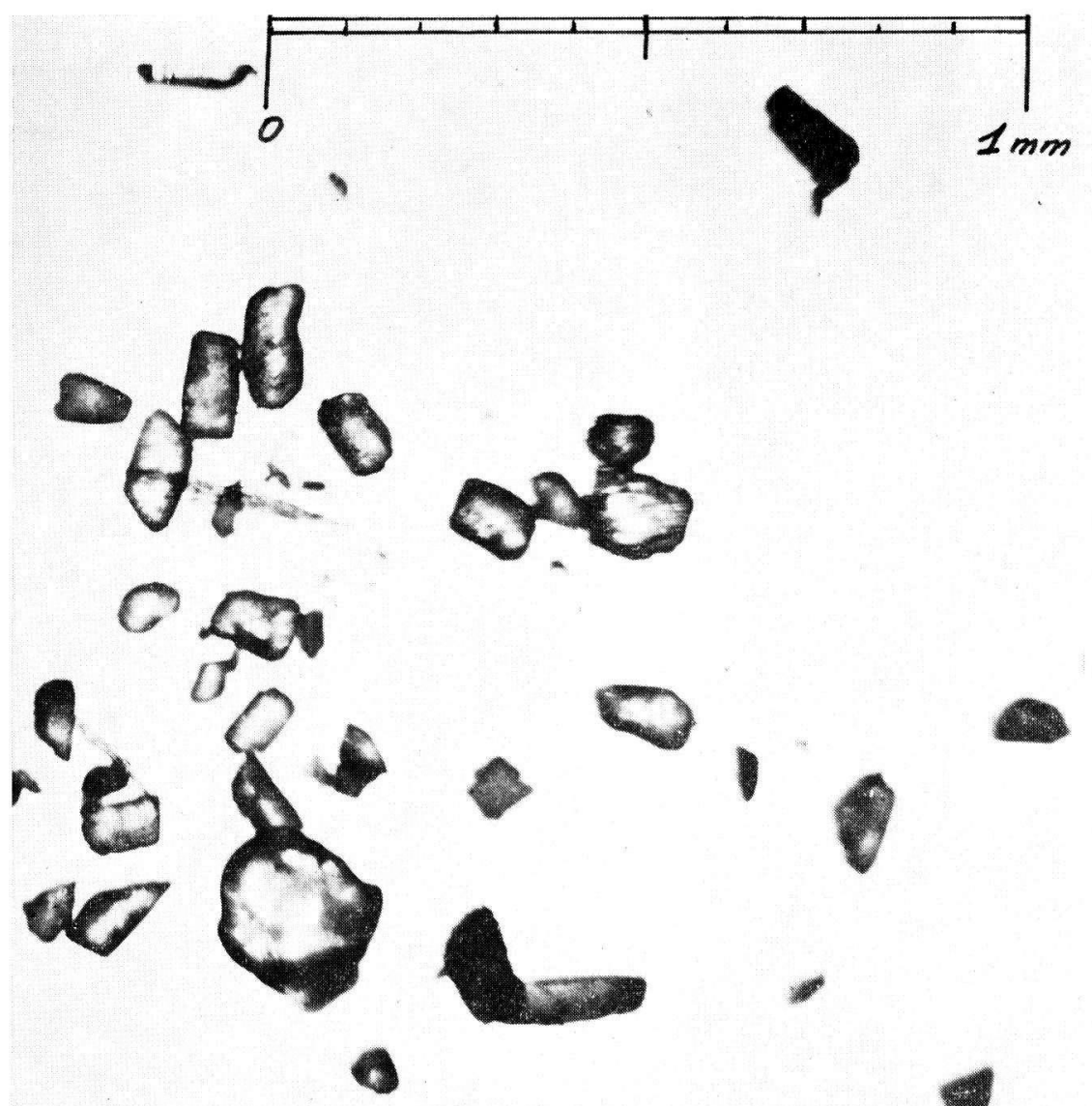

FIG. 4. - Photographie de cristaux extraits des vaisseaux du bois de peuplier (échelle: $\times 80$ )

FIG. 4. - Picture of crystals extracted of vessels of poplar wood (scale : $\times 80$ )

Nous pouvons procéder :

— soit à partir de coupes minces déchiquetées à l'aide d'un Ultra-Turrax qui est un broyeur possédant un stator et un rotor à dentures,

- soit à partir de plaquettes de bois réduites en sciure au broyeur à marteaux.

Le bois défibré et la sciure obtenus sont lavés et décantés plusieurs fois pour séparer les cristaux du végétal. 


\subsection{Analyses.}

Après avoir obtenu une quantité importante de cristaux, nous avons fait deux parts sur lesquelles nous avons pratiqué :

- une analyse chimique,

— une analyse cristallographique.

2.221. Analyse chimique. - Elle a montré que les cations qui composent ces cristaux purs sont :

$$
\begin{aligned}
& \mathrm{Ca}^{++}=93 \% \\
& \mathrm{Mg}^{++}=7 \% \\
& \mathrm{~K}^{+}=0 \%
\end{aligned}
$$

Lors des précédentes analyses, nous avions toujours observé simultanément dans les parties du bois qui présentaient des cristaux une forte proportion de $\mathrm{Ca}^{++}, \mathrm{Mg}^{++}$et $\mathrm{K}^{+}$.

Il ressort bien de cette nouvelle analyse que le $\mathrm{Ca}^{++}$provient des cristaux, ainsi qu'une faible partie du $\mathrm{Mg}^{++}$, alors que le reste des ions $\mathrm{Mg}^{++}$et la totalité du $\mathrm{K}^{+}$proviennent de sels solubles fixés par coprécipitation lors des dépôts.

\subsection{Identification des cristaux de carbonate.}

\section{Aspect morphologique :}

Examinés à la loupe binoculaire, les cristaux sont de forme et de couleur variables; leur taille est voisine de $1 / 10^{\mathrm{e}}$ de $\mathrm{mm}$.

On distingue :

- des cristaux en aiguilles, agglomérés suivant leur plus grande dimension, de couleur ocre clair,

- des cristaux isolés, de contours géométriques nets, de couleur blanche à ocre très clair, - de petits cristaux, sans forme géométrique définie, très colorés, de rouge à rouge sombre.

La couleur des cristaux provient vraisemblablement de produits organiques adsorbés sur les cristaux dans le bois de cœur.

\section{Examen radiocristallographique:}

Plusieurs cristaux blancs, blancs ocrés et rouges ont été examinés isolément; tous sont polycristallins.

L'identification sur monocristal se révélant impossible, nous avons effectué un diagramme de poudre. Les cristaux ont été séparés en deux lots :

1) cristaux en aiguilles,

2) cristaux isolés; les cristaux rouges étant en très faible quantité, il est probable qu'ils passent inaperçus sur un cliché Debye-Scherrer.

La correspondance parfaite entre les distances réticulaires de la calcite et des cristaux analysés prouvent de façon sûre qu'il s'agit bien de $\mathrm{CaCO}_{3}$ sous forme de calcite, et tend à montrer que le $\mathrm{Mg}^{++}$décelé par l'analyse chimique est trop faible pour pouvoir être mis en évidence par les spectres de diffraction des rayons X. 
TABLEAU 6

Résultats

Distance réticulaire. (Clichés Debye-Scherrer)

en $\AA$

\begin{tabular}{c|c|c|c}
\hline $\begin{array}{c}\text { Cristaux } \\
\text { en aiguilles } \\
(+)\end{array}$ & \multicolumn{2}{|c|}{ Petits cristaux } & Calcite \\
\hline & & & carte ASTM (5-0586) \\
3,85 & & & \\
3,03 & 3,85 & 3,83 & 3,86 \\
2,50 & 3,03 & 3,04 & 3,035 \\
$2,28_{8}$ & 2,49 & 2,47 & 2,495 \\
2,097 & $2,28_{0}$ & $2,28_{0}$ & 2,285 \\
1,916 & $2,09_{0}$ & $2,08_{3}$ & 1,095 \\
1,868 & 1,911 & 1,905 & 1,875 \\
1,606 & 1,866 & & 1,604 \\
1,525 & & & 1,525 \\
1,472 & & & 1,473 \\
1,439 & & & 1,440 \\
\hline
\end{tabular}

$(+)=$ cliché le plus intense.

\section{3. - DÉTERMINATION QUANTITATIVE}

\section{1. - Description de l'expérience sur Populus trichocarpa 'Fritzi-Pauley'.}

Dans notre précédente étude, nous avons fait apparaître sur trois sections transversales de peupliers Populus 'Robusta' l'existence d'un cerne-charnière qui sépare les zones aubier - bois de cœur du point de vue des teneurs en ions minéraux : phosphore, calcium, magnésium et potassium.

Une étude plus approfondie sur un Populus trichocarpa de $25 \mathrm{~m}$ de hauteur a été entreprise mètre par mètre; elle a permis de localiser les diverses pousses annuelles et d'individualiser dans chacune les accroissements annuels successifs pour confirmer ou infirmer l'existence de ce cerne-charnière tout au long de la tige.

Les méthodes d'analyses sont celles décrites dans notre précédente étude.

\section{2. - Analyses portant sur le phosphore}

Les résultats sont exprimés en partie par million de phosphore (PPM). Le tableau 7 et le diagramme (fig. $n^{\circ} 5$ ) portent en ordonnées les diverses pousses annuelles, en abscisses les accroissements annuels comptés de la moelle vers l'écorce. On obtient ainsi une image de la répartition du phosphore dans la tige. Chaque carré unitaire représente la concentration en ion phosphore du bois produit chaque année à chaque niveau. 
Remarque : Nous avons jugé opportun, compte tenu de l'aspect des diverses formes de phosphore dans l'arbre, d'exprimer le tableau des teneurs en P plutôt qu'en $\mathrm{P}_{2} \mathrm{O}_{5}$; cependant, les résultats primaires figurant en annexe sont exprimés en $\mathrm{P}_{2} \mathrm{O}_{5}$.

\section{Discussion :}

Les valeurs absolues des teneurs en phosphore permettent de distinguer 5 zones dans l'arbre :

1) le bois de cime.

Indifférencié et jeune ne comportant pas de bois de cœur et possédant des teneurs élevées de phosphore, caractéristiques d'un bois vivant, et supérieures à celles que l'on trouve dans l'aubier aux niveaux inférieurs. Il est intéressant de noter que la concentration en $\mathbf{P}$ atteinte au niveau de la pousse terminale est voisine de celle observée dans les tissus foliaires prélevés au niveau du houppier.

TABLEAU 7

Teneurs en ppm de phosphore dans P. trichocarpa 'Fritzi-Pauley'

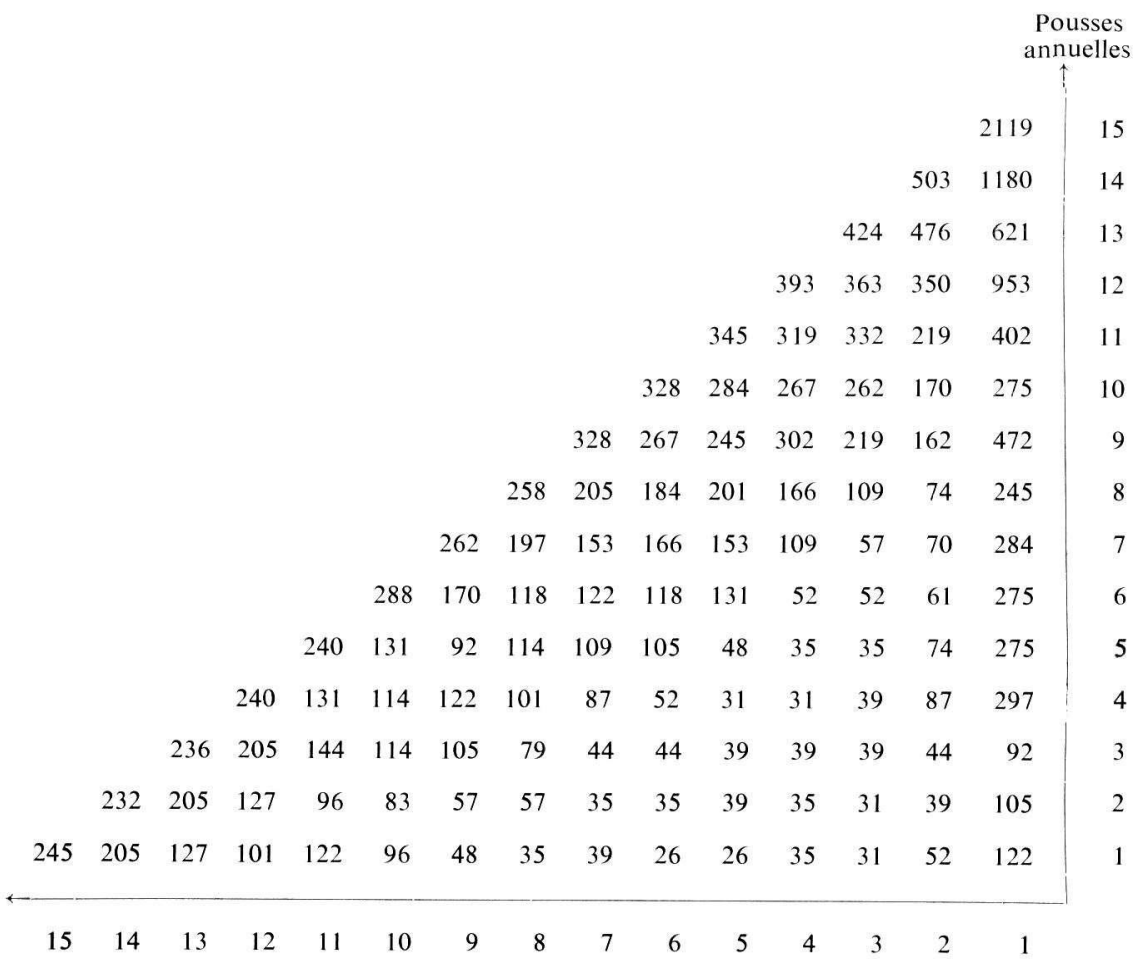

Accroissements annuels. 
Cette zone peut se subdiviser :

a) en une partie située entre 19 et 25 mètres, très riche en phosphore, comprenant les pousses annuelles 12, 13, 14 et 15 .

b) en une partie inférieure située entre 16 et 19 mètres oủ les teneurs sont intermédiaires entre celles de l'aubier et de la cime proprement dite et comprenant des pousses annuelles 9, 10 et 11 .

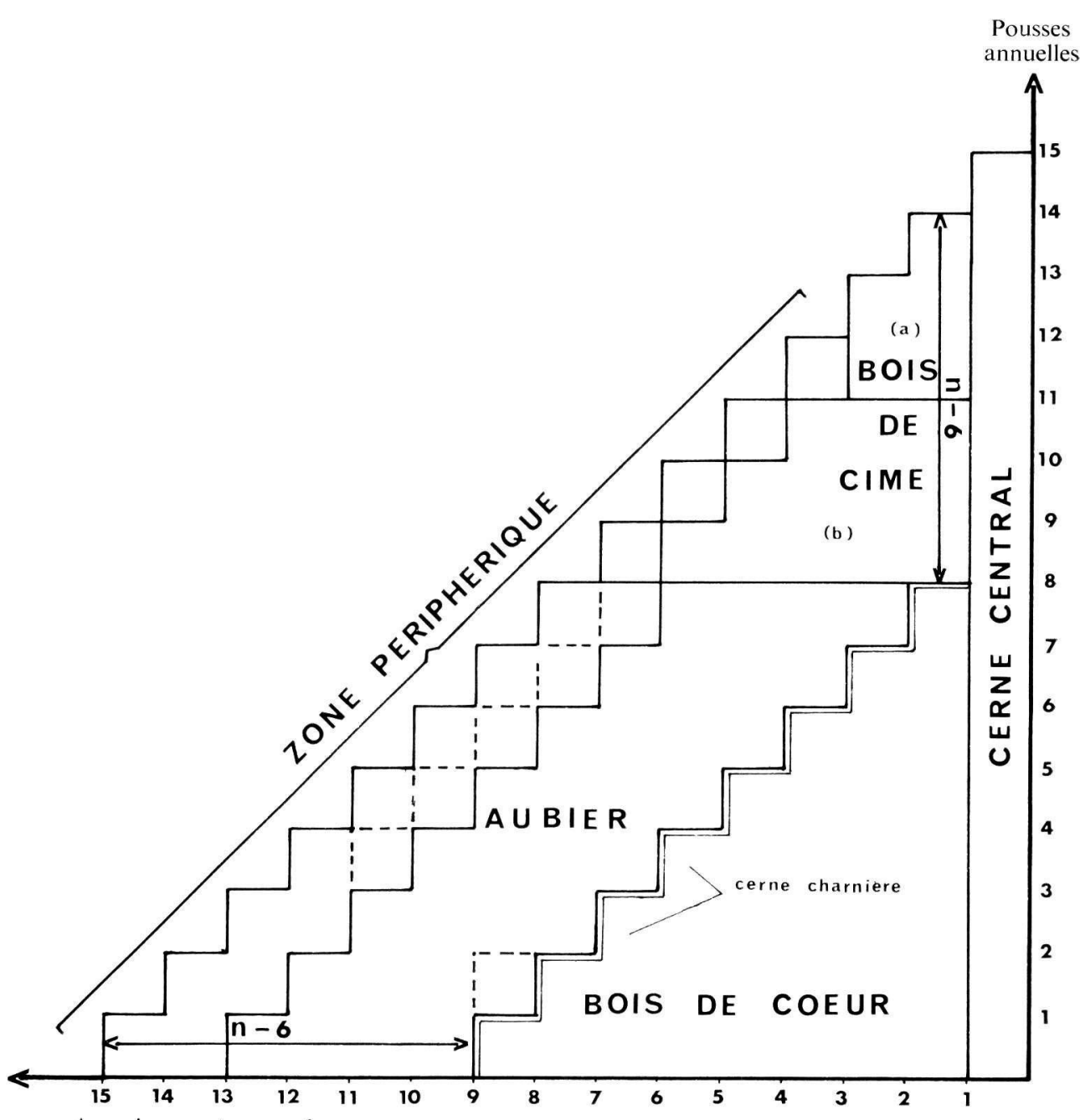

Accroissements annuels

FIG. 5. - Caractérisation des différentes zones dans un P. trichocarpa

'Fritzi-Pauley' en fonction des teneurs en phosphore

FIG. 5. - Caracterisation of different zones in P. trichocarpa

'Fritzi-Pauley' as a function of phosphorus contents 
On peut remarquer que ces deux zones sont celles qui possèdent le plus de branches vivantes de tout le houppier.

2) la zone périphérique.

située tout contre l'écorce qui subirait une imprégnation en $\mathrm{P}$ due à la proximité du cambium et qui se manifeste au maximum sur deux accroissements.

3) l'aubier.

où se réalise une transition sous forme d'un palier relativement constant des concentrations en phosphore entre la zone périphérique et le bois de cœur. Le nombre de cernes qui assurent ce passage ne varie d'ailleurs guère d'un niveau de prélèvement à l'autre.

L'aubier et la zone périphérique représentent sur toute la hauteur de l'arbre, 6 cernes annuels (ou moins dans la partie supérieure de la tige qui n'a pas encore 6 accroissements).

4) le bois de cour.

qui se caractérise par la chute brutale de concentration en phosphore au niveau d'un seul cerne-charnière et au-delà de cette limite par une concentration très faible jusqu'au cerne central non compris. Ces deux remarques indiquent que le bois de cœur ne participe plus aux échanges nutritifs dans l'arbre.

5) le cerne central.

qui entoure la moelle où le phosphore augmente brusquement. En effet, du sommet de l'arbre à 25 mètres jusqu'au niveau de 1 mètre, les teneurs demeurent, à quelques exceptions près, toujours plus élevées que dans le bois de cime, l'aubier et le bois de cœur situés aux mêmes niveaux.

Ceci pourrait être dû à une rémanence de la teneur exceptionnellement élevée du phosphore dans la pousse terminale de chaque année.

\section{4. - CONCLUSION}

\section{1. - Aspect qualitatif}

La présence ou l'absence de cristaux dans le bois de peuplier n'est pas un phénomène aléatoire. Après avoir examiné 28 espèces nous retrouvons, sans exception, l'opposition entre les espèces de la section Leuce sans cristaux et celles de toutes les autres sections Aigeiros, Tacamahaca, Leucoides et des hybrides entre sections qui en contiennent. Ce caractère qui semble lié à la génétique est indépendant de la nature du sol où se trouvent plantées ces espèces.

L'obtention de ces cristaux par broyage du bois est aisée suivant la méthodologie originale décrite dans le texte. Les analyses cristallographiques ont montré de façon sûre qu'il s'agissait de cristaux de carbonate de calcium.

En plus de leur intérêt pour la systématique du genre Populus et la physiologie de l'arbre, la présence de ces cristaux peut avoir une incidence sur la technologie de débit du bois. Ainsi, lors du sciage de certains peupliers de la plaine du Pô, le phénomène était tellement 
accentué dans ce cas particulier qu'il provoquait des dommages aux outils métalliques pendant le travail à l'usine. (communication personnelle ${ }^{1}$ ).

\section{2. - Aspect quantitatif et fonctionnel}

Le phosphore est un élément important pour les échanges nutritifs et énergétiques liés à l'élaboration des tissus végétaux. Une étude sur un Populus trichocarpa de $25 \mathrm{~m}$ de hauteur conduite mètre par mètre et cerne par cerne, qui a permis d'individualiser 208 échantillons de bois, fait apparaître 5 zones fonctionnelles différentes : le bois de cime, la zone périphérique, l'aubier, le bois de cour et le cerne central. Nous pouvons ainsi distinguer sur une même section le bois à forte teneur en phosphore - zone périphérique et aubier - du bois pauvre en phosphore - bois de cœur - le cerne central faisant exception avec une concentration élevée en phosphore, même au niveau inférieur où il est duraminisé.

Cette anomalie peut être considérée comme une rémanence des teneurs en phosphore exceptionnelles de chaque pousse annuelle. La chute brutale des concentrations observée sur 15 sections consécutives confirme indiscutablement l'existence d'un cerne-charnière pour les teneurs en ions phosphore.

Dans les études sur la duraminisation, nous posséderons un moyen analytique chimique et rationnel pour séparer l'aubier du bois de cœur, indépendamment de l'observation visuelle et empirique telle qu'on la pratique sur les autres espèces comme le Chêne, le Noyer ou le Merisier, où l'aubier et le duramen se distinguent par un changement de coloration du bois.

Reçu pour publication en novembre 1972.

\section{REMERCIEMENTS}

Nous adressons nos sincères remerciements à :

M. Онта, Chercheur à la Station Expérimentale des Forêts, Tokyo, stagiaire à la Station de la Qualité des Bois, pour les échantillons qu'il nous a fournis.

M. Duval, Technicien à la Station d'Amélioration des Arbres Forestiers pour la collecte d'échantillons.

M. Chmielewski de l'Instytut Badawczy Lesnictwa pour les échantillons fournis.

M. Laugier, Responsable du Service de Physico-chimie des Combustibles - Centre d'Études Nucléaires de Grenoble, qui a répondu favorablement aux demandes d'analyses que nous lui avons formulées.

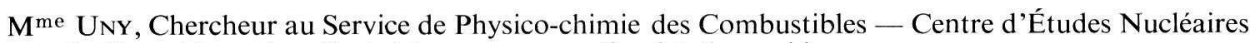
de Grenoble, qui a effectué les examens radiocristallographiques.

1. Communication de M. Walter Vivani, Directeur de l'Istituto nazionale per Piante da Legno Torino (Italia). 


\title{
SUMMARY
}

\section{COMPLEMENTARY STUDY OF THE PRESENCE OF CRYSTALS IN THE WOOD OF POPLARS. EXISTENCE OF FIVE ZONES RECOGNIZED BY THEIR PHOSPHORUS CONTENT.}

This work follows a study about the presence of calcium carbonate crystals in poplar wood (Annales des Sciences forestieres, 1972, 29, 1, pp. 67-105). After investigating on 28 species, we systematically find again the opposition between section Leuce, without crystals, and other sections : Aigeiros, Leucoides, Tacamahaca and hybrids, which contain crystals. This is not dependant of the soil on which the trees are grown.

These crystals can be easily obtained by grinding the wood and separated by decantation in water.

We must remark that, in same cases, their presence has an influence on wood technology.

Besides that, phosphorus has been dosed for every annual ring at every meter on the stem of a $P$. trichocarpa $c v$. "Fritzi-Pauley' $25 \mathrm{~m}$ high. This analysis shows five different zones : topwood, peripheric wood, sap wood, heart-wood and central ring.

These separations, related to tree physiology, will make studies on heart-wood formation easier.

\author{
ZUSAMMENFASSUNG \\ ERGANZENDE UNTERSUCHUNG ÜBER DIE VERTEILUNG \\ DER KALZIUMKARBONATKRISTALLE IM HOLZ DER PAPPELN. \\ NACHWEIS VON FÜNF ZONEN AN HAND IHRES PHOSPHORGEHALTES.
}

Die vorliegende Arbeit ergänzt die Untersuchungen über den Nachweis von Kalziumkarbonatkristallen im Holz der Pappeln (Annales des Sciences forestières, 1972, 29, 1, pp. 67-105).

Nach Untersuchung von 28 Arten bestätigt sich wiederum der bereits beschriebene Gegensatz zwischen den Arten der Sektion Leuce welche ausnahmslos keine Kristalle haben und allen übrigen Sektionen Aigeiros, Leucoides, Tacamahaca, und deren Hybride. Dieses Merkmal ist unabhängig vom Bodentyp des Standortes. werden.

Die Kristalle können sehr einfach durch mahlende Holzes und Dekantation im Wasser erhalten

Es muss hier erwähnt werden, dass die Kristalle in gewisse Fällen die technologische Sägeeigenschaft des Holzes beeinflussen können.

Desweiteren wurde an einem $25 \mathrm{~m}$ hohen Baum (Populus trichocarpa 'Fritzi-Pauley '). Eine Stammanalyse in 1 meter Sektionen durchgeführt und Jahrringweise auf den Gehalt an $\mathrm{CaCO}_{3}$ Kristallen untersucht. Es zeigte sich dabei, dass fünf funktional unterschiedliche Zonen festgestellt werden können : das Holz der Kronenregion, eine Randzone, das Splintholz, das Kernholz und der zentrale Jahrring. Diese Zonen die mit der Baumphysiologie zusammenhängen werden die Untersuchungen über die Kernholzbildung erleichtern.

\section{RIASSUNTO}

\section{STUDIO COMPLEMENTARE DELLA PRESENZA}

DEI CRISTALLI NEL LEGNO DEI PIOPPI.

ESISTENZA DI CINQUE ZONE FUNZIONALI RICONOSCIUTE

MEDIANTE LE LORO CONCENTRAZIONI IN FOSFORO.

Il presente rapporto completa uno studio pubblicato prima sulla messa in evidenza di cristalli di carbonato di calcio nel legno dei Pioppi (Annales des Sciences Forestières, 1972, 29, 1, 67-105).

Da allora abbiamo esaminati 28 specie nelle quali ritroviamo senza eccezione l'opposizione già segnalata fra le specie della sezione Leuce, prive di cristalli, e quelle delle sezioni Aigeiros, Leucoides, Tacamahaca e le loro ibride, che ne contengono. 
Questo carattere è indipendente della natura del suolo ove crescono queste specie.

Questi cristalli possono essere ottenuti tritturando fine il legno e separati col travaso nell'acqua.

Dobbiamo notare che in certi casi la loro presenza ha una influenza sulla tecnologia del legno.

Di più una analisi completa del fusto fu stata compiuta, metro a metro, e per ciascuna delle sezioni ad ogni metro, anello a anello, su uno P. trichocarpa 'Fritzi-Pauley' di 25 metri d'altezza. Mediante le concentrazioni in fosforo determinate per ciascuno degli anelli, abbiamo separati cinque zone funzionali differenti : il legno della cima, la zona periferica, l'alburno, il durame e l'anello centrale. del durame.

Questi distinzioni che riguardano la fisiologia dell'albero faciliteranno gli studi sulla formazione

\section{RÉFÉRENCES BIBLIOGRAPHIQUES}

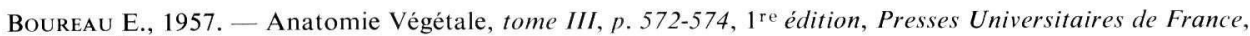
Paris.

Ellis E.-L., 1967. - Mineral, crystals, and heartwood. XIV e Congrès I.U.F.R.O., Section 41, Munich.

Gagnaire J., 1967. - Recherches sur la détection des pollutions radioactives par les végétaux. Thèse Docteur ès Sciences, Faculté des Sciences de l'Université de Grenoble.

JANIN G. et Clément A., 1972. - Mise en évidence de cristaux de carbonate de calcium dans le bois des peupliers. Conséquences sur la répartition des ions liés à la duraminisation. Ann. Sci. forest., 1972, 29 (I), 67-105.

Kennedy R. W., Sastry C. R. B., Barton G. M. et Ellis E. L., 1968. - Crystals in the wood of the genus Abies indigenous to Canada and the United States. Canadian Jouranl of Botany, vol. 46, $n^{\circ}$ 10, 1221-1228.

Meny L. et Champigny M., 1969. - Quelques applications métallurgiques du microscope électronique à balayage. J. Microscopic 8, 663-676.

Metcalfe C. R. et Chalk L., 1950. - Anatomy of Dicotyledons : leaves stem and wood in relation to taxonomy, with notes on economic use. Oxford, Clarendon Press, 2 Vol.

MiA A. J., 1969. - Light and electron microscope studies of crystalline substances in Acer Saccharum mineral stain. Wood Science, vol. $2, n^{\circ} 2,120-124$

Panshin A. J. et de Zeeuw C., 1964. - Textbook of wood technology, vol. 1, p. 154, 155, 159, seconde édition, Mc Graw-Hill Book Company, New York.

Philibert J., 1968. - La microscopie électronique à balayage et ses applications métallurgiques. Revue de Physique Appliquée, tome III, décembre, p. 325-330.

Polge H., 1966. - Établissement des courbes de variation de la densité du bois par exploration densitométrique de radiographies d'échantillons prélevés à la tarière sur des arbres vivants. Applications dans les domaines technologique et physiologique. Thèse Docteur ès Sciences Appliquées, Faculté des Sciences de l'Université de Nancy.

Polge H., 1967. - Première appréciation de la qualité du bois de Populus trichocarpa cultivé dans le Populetum de Velaine. Document à usage interne, Station de Recherches sur la Qualité des Bois du C.N.R.F.

Ralston C. W. et Prince A. B., 1963. - Accumulation of dry matter and nutrients by Pine and Hardwood forests in the Lower Piedmont of North California. Forest-Soil relationships in North America, p. 76-94, Youngberg, Corvallis.

RECORD S. J., 1927. - Occurrence of calcium carbonate deposits in woods. Tropical woods, $n^{\circ}$ 12, p. 22-26. 
Tableau des teneurs en phosphore: $\mathrm{P}_{2} \mathrm{O}_{5} \%$ dans P. Trichocarpa 'Fritzi Pauley'

Hauteur du prélèvement en mètres

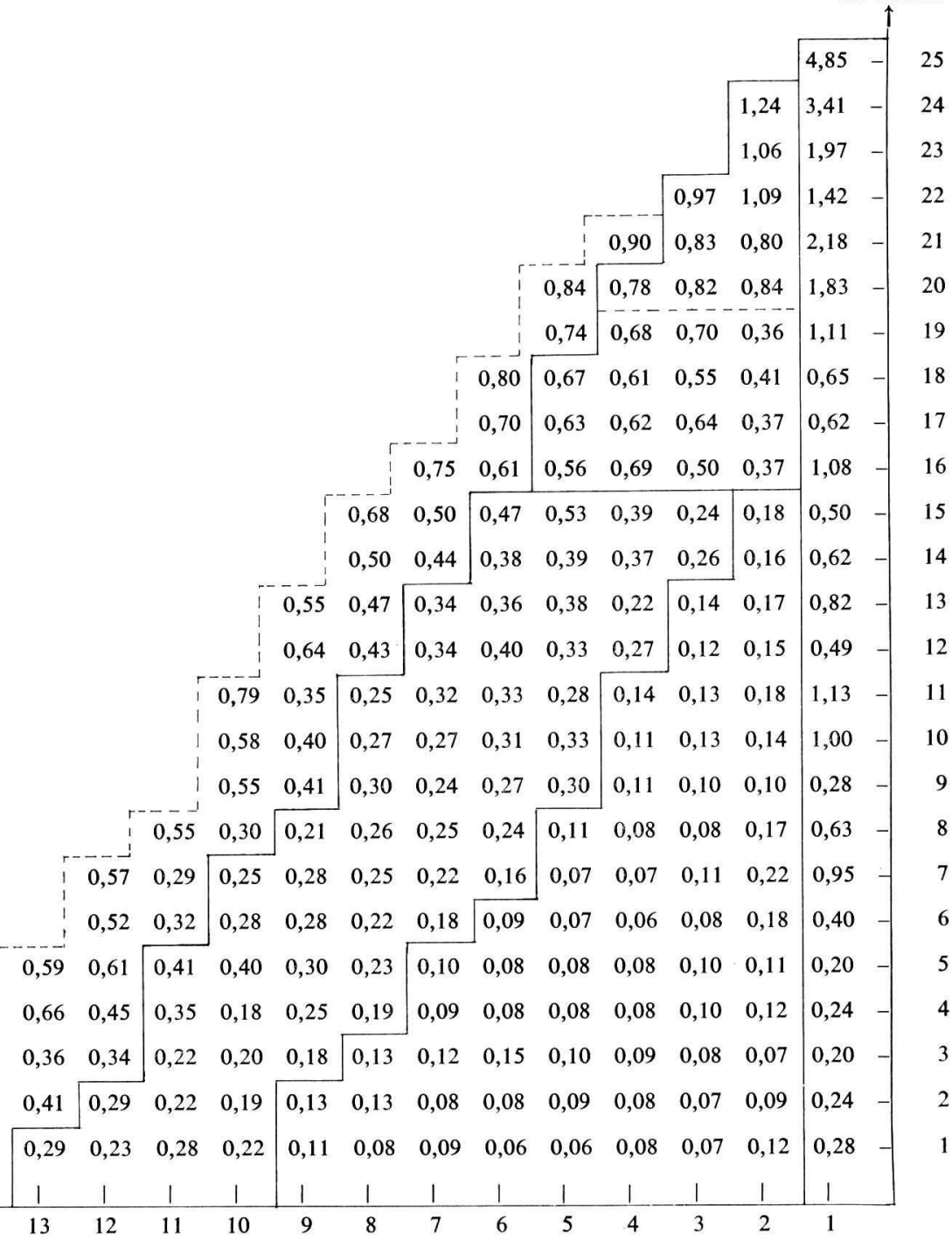

No du cerne de la moelle vers l'écorce 